\title{
The relationship between pigment epithelial detachment and visual outcome in neovascular age-related macular degeneration and polypoidal choroidal vasculopathy
}

\author{
Kai Xiong Cheong $\mathbb{1}^{1} \cdot$ Dilraj Singh Grewal ${ }^{2} \cdot$ Kelvin Yi Chong Teo ${ }^{1,3} \cdot$ Alfred Tau Liang Gan $^{1} \cdot$ Glenn Jay Jaffe $^{2} \cdot$ \\ Gemmy Chui Ming Cheung ${ }^{1,3}$
}

Received: 23 October 2019 / Revised: 31 January 2020 / Accepted: 31 January 2020 / Published online: 11 February 2020

(c) The Author(s), under exclusive licence to The Royal College of Ophthalmologists 2020

\begin{abstract}
Background/objectives To compare the detailed optical coherence tomography (OCT)-based morphological parameters of pigment epithelial detachment (PED) in eyes presenting with neovascular age-related macular degeneration (nAMD) and polypoidal choroidal vasculopathy (PCV), and to assess whether these PED-associated parameters influence 1-year visual outcomes.

Subject/methods We analysed images from a prospective observational study of treatment-naive Asian participants with nAMD or PCV. An independent reading centre graded baseline morphological features of PED on spectral-domain OCT, including greatest height, greatest width, greatest volume, morphology (predominantly dome shaped versus peaked), presence of retinal pigment epithelium (RPE) tear and cholesterol bands. The influence of these baseline features on 12 months best corrected visual acuity (BCVA) was evaluated.

Results Seventy-eight eyes of 78 participants with PED were studied. In total, 40 (51.3\%) participants had nAMD and 38 (48.7\%) had PCV. Eyes with PCV, compared with nAMD, had PED of greater height $(455.9 \mu \mathrm{m}$ versus $389.9 \mu \mathrm{m} ; P=$ $0.035)$ and had higher prevalence of RPE tear $(22.9$ versus $5.3 \% ; P=0.041)$. In the multivariate analysis, only baseline BCVA was significantly associated with month 12 BCVA, but none of the PED-associated OCT parameters at baseline influenced month 12 BCVA.

Conclusions Despite the differences in PED height and prevalence of RPE tear between nAMD and PCV, none of these PED morphological factors on OCT at baseline significantly influenced visual outcome at 12 months.
\end{abstract}

\section{Introduction}

Age-related macular degeneration (AMD) is a major cause of severe and irreversible visual impairment [1-3]. The outcome of treatment of AMD has improved with the introduction of anti-vascular endothelial growth factor (VEGF) therapy [4, 5]. Favourable anatomical and

Gemmy Chui Ming Cheung

gemmy.cheung.c.m@snec.com.sg

1 Singapore Eye Research Institute, Singapore National Eye Centre, Singapore, Singapore

2 Department of Ophthalmology, Duke University, Durham, NC, USA

3 Ophthalmology \& Visual Sciences Academic Clinical Program (Eye ACP), Duke-NUS Medical School, Singapore, Singapore functional response up to 2 years have been reported [6, 7]. However, long-term results are variable and significant heterogeneity has been described. The variability in treatment response can be partially accounted for by differences in macular morphology [8].

Considerable effort has been expended to identify, characterise and validate morphological features of significant prognostic value and functional importance on optical coherence tomography (OCT). One of these features, pigment epithelial detachment (PED), is observed frequently in eyes with neovascular AMD (nAMD) and occurs in up to $80 \%$ of such eyes depending on the study [9-11]. PED is also a very common feature in polypoidal choroidal vasculopathy (PCV), an AMD variant with a high prevalence in Asian populations [12].

Previous studies have reported inconsistent results regarding the relationship between PED and visual outcomes [13-34]. Whilst some studies have associated 
the presence of PEDs [15-17, 22, 31], or larger PEDs with less favourable visual outcomes [25, 28, 33], others have reported no significant relationships $[13,14,18,20,21$, $23,24,27,30,32]$. In the VIEW study for example, $80.1 \%$ of eyes had PED at baseline, but resolved in only $38 \%$ [17]. Furthermore, the presence of PED at baseline was predictive of a negative outcome at 48 weeks [17].

Recently, we evaluated the influence of multiple morphological features on visual outcomes in Asian patients with nAMD and PCV [12]. In that cohort, PED was a common morphological feature that was present in 85.4 and $91.1 \%$ in non-PCV eyes and PCV eyes, respectively [12]. However, the presence of PED at baseline did not significantly influence visual acuity at month 12 .

To date, although various studies have investigated the prognostic value of baseline PED, there is limited data on whether specific PED characteristics influence visual acuity outcomes. Since PED was highly prevalent in our cohort, we hypothesised that the detailed individual features of PED may be important to predict treatment outcome. Recognising the need to characterise PEDs in nAMD and $\mathrm{PCV}$, and to not just consider its presence or absence, the primary aim of this study was to assess whether specific baseline PED morphological features on OCT would influence 12-month visual acuity in an Asian population. A second aim was to determine whether PED morphology and visual acuity outcomes differ in eyes with nAMD and PCV.

\section{Methods}

\section{Study design and population}

This study represents an analysis of clinical and imaging data acquired from participants with nAMD and PCV who were recruited prospectively under the Phenotyping Asian Macular Diseases study, for which the detailed methodology was published previously [35]. Briefly, the Phenotyping Asian Macular Diseases study is an ongoing, prospective, observational clinical study of treatment-naive Asian participants in Singapore with exudative maculopathy signs secondary to typical nAMD or PCV. All patients in this ongoing study undergo a complete ophthalmic examination and investigations according to a standardised protocol. This study is a subgroup analysis of the participants who presented with PED at baseline from the previous analysis [12]. Written informed consent was provided by all participants before participation in the study. The study was approved by the institutional ethics committee (Protocols R697/47/2009 and R1338/24/2016) and conformed to the tenets of the Declaration of Helsinki.

\section{Study assessment}

The outcome measure of interest was best corrected visual acuity (BCVA) at month 12 , which was determined with a Snellen chart and converted to LogMAR equivalent.

Spectral-domain OCT (SD-OCT) images at baseline prior to treatment were obtained using the Spectralis OCT (Heidelberg Engineering, Heidelberg, Germany). SD-OCT raster scans with enhanced depth imaging were acquired on a $30^{\circ} \times 20^{\circ}(8.7 \mathrm{~mm} \times 1.9 \mathrm{~mm})$ macular region centred on the fovea, in the high-speed mode, with a minimum of 25 B-scans per volume scan. Each B-scan was averaged (Automatic Real-time Tracking (ART) mode) using 7-9 frames, and the distance between consecutive B-scans ranged from 233 to $244 \mu \mathrm{m}$.

\section{OCT image grading and definitions}

The analysis of qualitative and quantitative morphological features on SD-OCT scans was performed by trained readers at the Duke Reading Center. They were masked to treatment and BCVA, and the grading was performed according to a pre-specified protocol.

The definition of a PED in this study was a focal elevation of the reflective retinal pigment epithelium (RPE) band over an optically clear or moderately reflective space, either higher than $200 \mu \mathrm{m}$ or wider than $400 \mu \mathrm{m}$.

The PED OCT morphological parameters of interest in this study were greatest height, greatest width, greatest volume, morphology (predominantly dome shaped versus peaked), the presence of RPE tear and the presence of cholesterol bands.

The height and width of the PED at baseline were measured by two masked readers as the distance between the inner surface of the Bruch's membrane and the outer surface of the RPE using the caliper function on the Spectralis Heidelberg Eye Explorer (HEYEX) image management platform (version 1.10.4.0) The volume of the PED was approximated as the greatest height of the PED $\times 0.5$ (greatest width of the PED).

Peaked PED was defined as a steep sharp peak-like elevation of the RPE with underlying moderate reflectivity within the peak [36]. Dome-shaped PED was defined as a round and smooth contour of the elevation of the RPE [36]. RPE tear was defined as a disruption of the RPE monolayer, which can vary from a microscopic defect to a large defect with an irregular contour of the retracted RPE, often with hyper-reflectivity from duplication of the RPE and increased signal transmission in the areas with an absence of the RPE [37, 38]. Cholesterol bands were defined as the presence of layered hyperreflective lines in the sub-RPE space [39]. 


\section{Treatment}

Treatment was determined by the attending retinal specialist. Typically, participants were treated with anti-VEGF therapy when there was subretinal or intraretinal fluid or haemorrhage associated with active disease. For PCV, in cases where the polypoidal lesions were small and when VA was good, anti-VEGF monotherapy was used. Photodynamic therapy (PDT) was given as an adjunct to antiVEGF therapy for extensive or subfoveal polypoidal lesions. Subsequent re-treatment was based on VA, clinical examination and OCT morphology. A variety of regimens were used including pro re nata and treat and extend.

\section{Statistical analysis}

Statistical analyses were performed using the Stata Statistical Software: Release 15 (StataCorp LLC, College Station, TX, USA). Continuous variables were summarised using means and standard deviations (SD), and categorical variables with counts and percentages. We compared the PED morphological parameters and BCVA between nAMD and PCV using the Mann-Whitney $U$-test for continuous variables, and Fisher's exact test for categorical variables.

A univariate linear regression analysis was performed to determine possible associations between each PED morphological parameter and month 12 BCVA. We then constructed a multiple regression model that comprised factors, which were found to be significantly associated with month 12 BCVA in a previous study or that were deemed to be clinically important
[12]. This base model included age, gender, cardiovascular disease, PCV phenotype, number of anti-VEGF injections, number of PDT treatments, smoking status, baseline BCVA and central retinal thickness (CRT). Multiple imputation was performed to address missing variable data, assuming a missing at random mechanism. We then added the PED morphological parameters to the base model and assessed changes to the coefficient of determination $\left(R^{2}\right)$ to see if any of the PED morphological parameters caused additional variation in month 12 BCVA over and above that caused by the base model factors. A joint Wald test of beta coefficients was also conducted to determine if the PED morphological parameters in combination improved overall model fit. A $P$ value $<0.05$ was considered statistically significant.

\section{Results}

We included 78 eyes of 78 participants with a baseline PED. In total, 40 (51.3\%) participants had nAMD and 38 (48.7\%) had PCV.

At baseline, the overall mean age of participants was $70.4 \pm 9.1$ years. Participants with nAMD were older compared with participants with PCV $(74.0 \pm 7.5$ years versus $66.7 \pm 9.3$ years, $P=0.001)$. In total, $34(43.6 \%)$ of the participants were female. The overall BCVA was $0.79 \pm$ 0.51 LogMAR units, and the overall CRT was $471.2 \pm$ $175.0 \mu \mathrm{m}$. Gender, BCVA and CRT were not significantly different between the nAMD and PCV groups at baseline. The baseline characteristics are summarised in Table 1.

Table 1 Baseline data.

\begin{tabular}{|c|c|c|c|c|}
\hline & \multicolumn{3}{|c|}{ Mean $\pm \mathrm{SD}$ or $n(\%)$} & \multirow[t]{2}{*}{$P$} \\
\hline & $\begin{array}{l}\text { Overall } \\
n=78 \text { eyes }\end{array}$ & $\begin{array}{l}\text { nAMD } \\
n=40 \text { eyes }\end{array}$ & $\begin{array}{l}\text { PCV } \\
n=38 \text { eyes }\end{array}$ & \\
\hline Age (years) & $70.4 \pm 9.1$ & $74.0 \pm 7.5$ & $66.7 \pm 9.3$ & 0.001 \\
\hline Female gender & $34(43.6)$ & $16(40.0)$ & $18(47.4)$ & 0.648 \\
\hline LogMAR BCVA & $0.79 \pm 0.51$ & $0.71 \pm 0.47$ & $0.87 \pm 0.54$ & 0.159 \\
\hline CRT $(\mu \mathrm{m})$ & $471.2 \pm 175.0$ & $466.7 \pm 162.1$ & $476.4 \pm 191.2$ & 0.936 \\
\hline \multicolumn{5}{|l|}{ PED parameters } \\
\hline Height $(\mu \mathrm{m})$ & $421.6 \pm 510.0$ & $389.9 \pm 609.1$ & $455.9 \pm 380.6$ & 0.035 \\
\hline Width $(\mu \mathrm{m})$ & $1952.6 \pm 1389.3$ & $1858.2 \pm 1159.6$ & $2054.7 \pm 1611.7$ & 0.931 \\
\hline Volume $\left(\times 10^{3} \mu \mathrm{m}^{3}\right)$ & $462.6 \pm 601.7$ & $383.6 \pm 500.4$ & $545.7 \pm 689.7$ & 0.472 \\
\hline \multicolumn{5}{|l|}{ Morphology $\mathrm{y}^{\mathrm{a}}$} \\
\hline Dome-shaped & $24(32.0)$ & $10(27.0)$ & $14(36.8)$ & 0.328 \\
\hline Peaked & $51(68.0)$ & $27(73.0)$ & $24(63.2)$ & 0.813 \\
\hline RPE tear & $10(13.7)$ & $2(5.3)$ & $8(22.9)$ & 0.041 \\
\hline Cholesterol bands & $2(2.6)$ & $2(5.0)$ & $0(0.0)$ & 0.494 \\
\hline
\end{tabular}

$S D$ standard deviation, $n$ number.

Statistically significant $p<0.05$ values are in bold.

${ }^{\text {a }}$ Total of 75 eyes. 
Table 2 Univariate analysis of month 12 BCVA against PED morphological factors.

\begin{tabular}{lcll}
\hline PED Parameters on OCT & Beta $(95 \% \mathrm{CI})$ & $P$ & $R^{2}$ \\
\hline Height $($ per $\mathrm{SD}=510.0 \mu \mathrm{m})$ & $0.08(-0.06,0.21)$ & 0.25 & 0.02 \\
Width $($ per $\mathrm{SD}=1389.3 \mu \mathrm{m})$ & $0.08(-0.06,0.21)$ & 0.26 & 0.02 \\
Volume $\left(\right.$ per $\mathrm{SD}=601.7 \times 10^{3}{\left.\mu \mathrm{m}^{3}\right)}$ & $0.10(-0.03,0.23)$ & 0.15 & 0.03 \\
Morphology & & & \\
$\quad$ Dome-shaped & $-0.22(-0.50,0.07)$ & 0.14 & 0.03 \\
$\quad$ Peaked & $0.17(-0.11,0.44)$ & 0.23 & 0.02 \\
RPE tear & $0.15(-0.24,0.54)$ & 0.46 & 0.01 \\
Cholesterol bands & $0.60(-0.23,1.43)$ & 0.15 & 0.03 \\
\hline
\end{tabular}

CI confidence interval.

\section{PED in $\mathrm{nAMD}$ and PCV}

The nAMD and PCV PED morphological parameters at baseline were first compared (see Table 1). Eyes with PCV, compared with eyes with nAMD, had PEDs of greater height $(455.9 \pm 380.6 \mu \mathrm{m}$ versus $389.9 \pm 609.1 \mu \mathrm{m}, \quad P=$ $0.035)$ and had higher prevalence of RPE tear (22.9 versus $5.3 \%, P=0.041)$. There were no significant differences between nAMD and PCV groups for the other PED-related morphological parameters.

\section{Treatment exposure and BCVA at month 12}

The mean number of anti-VEGF injections received from baseline to month 12 was $5.1 \pm 2.9$, with no significant difference in the number between nAMD and PCV groups. The overall mean number of PDT treatments was $0.3 \pm 0.5$. Among the $27(34.6 \%)$ patients who had at least 1 PDT treatment, the mean number of PDT treatments was $1.4 \pm 0.6$.

Over 12 months, BCVA improved in the PCV group $(-0.26 \pm 0.59$ LogMAR units) and worsened slightly in the nAMD group $(0.09 \pm 0.37$ LogMAR units). The month 12 BCVA for the nAMD and PCV groups was not significantly different $(0.79 \pm 0.58$ versus $0.62 \pm 0.59, P=0.136)$.

\section{Association of PED morphological parameters with month 12 BCVA}

In the univariate linear regression analysis (see Table 2), none of the PED OCT morphological parameters at baseline significantly influenced month 12 BCVA. Next, in the multivariate linear regression analysis, we added the PED morphological parameters to the base model, which comprised factors including age, gender, nAMD/PCV diagnosis, baseline BCVA, baseline CRT and treatment exposure to determine if the inclusion of PED morphological parameters would significantly improve the predictive value of the combined model (see Table 3). Only baseline BCVA (per line) $($ Beta $=0.06 ; P<0.001)$ was significantly associated with month $12 \mathrm{BCVA}$, with a model $R^{2}$ of 0.47 . None of the
Table 3 Multivariate analysis of month 12 BCVA against PED morphological factors.

\begin{tabular}{lccc}
\hline & Beta $(95 \%$ CI $)$ & $P$ & $R^{2}$ \\
\hline Base model & & 0.5 \\
Age (per 10 years) & $0.08(-0.05,0.21)$ & 0.236 \\
Gender female & $-0.05(-0.33,0.23)$ & 0.718 \\
BCVA (per line) & $0.06(0.04,0.09)$ & $<\mathbf{0 . 0 0 1}$ \\
CRT (per SD = 175.0 $\mu \mathrm{m})$ & $0.08(-0.05,0.21)$ & 0.203 \\
Cardiovascular disease & $0.30(-0.08,0.69)$ & 0.121 \\
Smoker & $0.10(-0.21,0.40)$ & 0.538 \\
PCV phenotype & $-0.20(-0.49,0.09)$ & 0.170 \\
Number of anti-VEGF injection (per & $-0.00(-0.04,0.04)$ & 0.956 \\
injection) & & & \\
Number of PDT treatment (per & $0.03(-0.26,0.32)$ & 0.843 \\
treatment) & & 0.678 & 0.5 \\
Base model + PED parameters & & & \\
Height (per SD $=510.0 \mu \mathrm{m})$ & $0.08(-0.03,0.18)$ & 0.173 & 0.5 \\
Width (per SD $=1389.3 \mu \mathrm{m})$ & $-0.06(-0.18,0.07)$ & 0.371 & 0.5 \\
Volume (per SD $\left.=601.7 \times 10^{3} \mu \mathrm{m}^{3}\right)$ & $-0.002(-0.13,0.13)$ & 0.981 & 0.5 \\
Morphology & & & \\
$\quad$ Dome-shaped & $0.04(-0.21,0.29)$ & 0.734 & 0.5 \\
$\quad$ Peaked & $-0.07(-0.31,0.17)$ & 0.541 & 0.5 \\
RPE tear & $0.16(-0.18,0.51)$ & 0.353 & 0.5 \\
Cholesterol bands & $-0.24(-1.09,0.61)$ & 0.575 & 0.5 \\
\hline CI & & &
\end{tabular}

CI confidence interval.

Statistically significant $p<0.05$ values are in bold.

PED OCT morphological parameters at baseline further influenced month 12 BCVA beyond that of the base model. The overall model $R^{2}$ increased marginally to 0.51 .

\section{Discussion}

The challenges of managing nAMD and PCV underscore the crucial role of imaging markers in a disease that has a global burden, increasing prevalence, and which requires extensive periods of treatment and follow-up. In this study, we found that eyes with PCV had PEDs of greater height and had a higher prevalence of RPE tear at baseline compared with eyes with nAMD. Despite these differences, none of these PED morphological factors on OCT at baseline significantly influenced visual outcome at 12 months.

In this study, the difference in height between PEDs in eyes with PCV and nAMD is consistent with that reported in previous studies [40, 41]. Also, the increased prevalence of RPE tear in eyes with PCV may be accounted for by the greater PED height. The increased risk of RPE tear in the presence of large PEDs is well recognised and is now accepted as largely related to the natural history of PED [42]. In addition to PCV, type 3 neovascularisation is another lesion subtype that often presents with large PEDs [43]. Whether there is an increased risk of RPE tear in PEDs related to PCV specifically has not been demonstrated. 
Despite these differences in PED characteristics in this study, BCVA at month 12 was similar between the two groups. Although the improvement in BCVA was larger in the PCV group compared with the nAMD group, once we adjusted for baseline BCVA and age, the difference becomes insignificant. None of the baseline PED morphological parameters on OCT further influenced month 12 BCVA beyond that of the base model.

There have been many studies, which have investigated the relationship between PEDs at baseline and final visual outcomes and have reported inconsistent relationships [13-34]. Generally, post-hoc analyses of the major prospective treatment trials had focused solely on the presence or absence of PED [13-18, 20, 21] In the post-hoc analyses of the EXCITE [14], MONT BLANC [18] and PRONTO studies [20, 21], the presence of PED at baseline did not significantly predict BCVA gains at 12 months. PED height at baseline was also analysed in the post-hoc analysis of the HARBOR study, and significant vision gains of 7.9-9.0 letters were achieved regardless of the height [13]. In contrast, the post-hoc analyses of the VIEW 1 and 2 studies suggested that the presence of PED at baseline had a small negative impact on BCVA $[15,17]$. Waldstein et al. reported 1.88 letters less BCVA improvement from baseline to week 52 [15]. Schmidt-Erfurth et al. reported a loss of 2.27 and 2.12 letters at 12 and 52 weeks, respectively [17]. Another post hoc analysis of the EXCITE study found that PED predicted a poor visual outcome only if combined with intraretinal cysts or subretinal fluid [16].

The studies which had attempted to use quantitative morphological parameters within the PED like height, width, area and volume are largely retrospective in nature [24, 25, 27, 28, 30, 33]. Similarly, a few studies reported weak to moderate negative correlations between the PED parameters at baseline and final BCVA [25, 28, 33]. Many others, however, reported that these parameters have no predictive value at all [24, 27, 30].

Overall, it is difficult to compare the various studies due to large study design differences that include the study population size, type of treatment, re-treatment criteria, type of OCT devices and choice of PED parameters [13-34]. Notably, the definition of a PED is varied. Some studies, particularly the prospective trials, have attempted to define a PED by qualitatively describing the OCT appearance of PED [13], or by specifying minimum heights or widths [14-17, 19, 22, 24, 25], relative proportions to the choroidal neovascularisation lesion on OCT [30], or areas on fundus fluorescein angiography [26, 29]. Many others did not have any specific definition for a PED. There may also be a difference in response depending on whether the PED is predominantly vascularised or serous. A few studies have demonstrated worse visual acuity, or less visual acuity improvement in fibrovascular and vascularised PEDs compared with serous and avascular PEDs after treatment [19, 26, 29]. Furthermore, the range of PED prevalence in these studies is large (18-90\%) [13-34]. Most of the prospective treatment trials, except for the HARBOR study, used time-domain OCT (TD-OCT) [14-18, 20, 21]. Results of studies which used TD-OCT may not accurately reflect treatment outcomes in modern care evaluated using SDOCT.

There was an unexpectedly high prevalence of eyes with "peaked PED" in the nAMD group. The morphology (predominantly dome shaped versus peaked) in PED was not significantly different between nAMD and PCV in this study. This finding was unexpected because the presence of a peaked PED is commonly associated with the presence of a polypoidal lesion beneath the Bruch's membrane in PCV [44]. DeSalvo et al. noticed this feature in $91.9 \%$ of the eyes with PCV, whereas $7.1 \%$ of the eyes with occult choroidal neovascularisation showed this feature [45]. This observation highlights the limitation of using individual OCT parameters to differentiate PED in PCV from other entities. Indocyanine green angiography remains the gold standard to diagnose PCV and should be performed if available. It is possible that using higher resolution OCT scans with reduced interscan spacing and incorporating other OCT tomographic signs that have been described in PCV may help improve the sensitivity of OCT.

Hyperreflective bands under PED, thought to represent cholesterol bands, have recently been recognised as a potential biomarker for treatment response [39]. This finding is attributed to chronic exudation in type 1 choroidal neovascularisation and the prevalence reportedly ranges from 4.2 to $15.6 \%$ [39]. In the current study, cholesterol bands were only observed in two eyes $(2.6 \%)$ with nAMD, and in none of the eyes with PCV. We are therefore not able to conclude the significance of this feature in the current cohort due to the low prevalence.

The strengths of this study include the use of a standardised image grading protocol which allowed a detailed and comprehensive assessment of PED morphological parameters on OCT that enabled us to compare PED features in eyes with nAMD to those with PCV, and to evaluate the influence of individual morphological factors on BCVA, with adjustment made for other covariates in the baseline model. The limitations of our study include the relatively small number of cases in the subgroup analysis and a short follow-up period of 12 months. It will also be interesting to explore whether other clinically meaningful end points of visual function such as distortion and microperimetry might give alternative results.

In summary, this study suggests that eyes with PCV have PEDs of greater height and have higher prevalence of RPE tear compared with eyes with nAMD. However, the PED characteristics at baseline have little value in 
prognosticating VA outcome. We believe that this study represents one of the most comprehensive and detailed phenotyping projects of PED in Asian nAMD and PCV. These data will add further to our current understanding of factors that influence treatment outcomes in the antiVEGF era.

\section{Summary}

\section{What was known before}

- Previous studies have reported inconsistent results regarding the relationship between PED and visual outcomes.

- Whilst some studies have associated the presence of PEDs, or larger PEDs with less favourable visual outcomes, others have reported no significant relationships.

- There is limited data on whether specific PED characteristics influence visual acuity outcomes.

\section{What this study adds}

- Despite the differences in PED height and prevalence of RPE tear between nAMD and PCV, none of the investigated PED morphological factors on OCT at baseline significantly influenced visual outcome at 12 months.

Funding This study is supported by the Duke/Duke-NUS Research Collaborations Grant: Duke/Duke-NUS/RECA(Pilot)2016/0020, the Biomedical Research Council Singapore Grant: SPF2014/002 and the National Medical Research Council Open Fund Large Collaborative Grant: NMRC/LCG/004/2018.

\section{Compliance with ethical standards}

Conflict of interest The authors declare that they have no conflict of interest.

Publisher's note Springer Nature remains neutral with regard to jurisdictional claims in published maps and institutional affiliations.

\section{References}

1. Lim LS, Mitchell P, Seddon JM, Holz FG, Wong TY. Age-related macular degeneration. Lancet. 2012;379:1728-38.

2. Wong WL, Su X, Li X, Cheung CM, Klein R, Cheng CY, et al. Global prevalence of age-related macular degeneration and disease burden projection for 2020 and 2040: a systematic review and meta-analysis. Lancet Glob Health. 2014;2:e106-16.

3. Kawasaki R, Yasuda M, Song SJ, Chen SJ, Jonas JB, Wang JJ, et al. The prevalence of age-related macular degeneration in
Asians: a systematic review and meta-analysis. Ophthalmology. 2010;117:921-7.

4. Rosenfeld PJ, Brown DM, Heier JS, Boyer DS, Kaiser PK, Chung $\mathrm{CY}$, et al. Ranibizumab for neovascular age-related macular degeneration. N Engl J Med. 2006;355:1419-31.

5. Comparison of Age-related Macular Degeneration Treatments Trials Research Group, Martin DF, Maguire MG, Fine SL, Ying GS, Jaffe GJ, et al. Ranibizumab and bevacizumab for treatment of neovascular age related macular degeneration: two-year results. Ophthalmology. 2012;119:1388-98.

6. Cheung CM, Chen H. Hidden messages in optical coherence tomography: looking beyond fluid. Ann Eye Sci. 2018;3:56.

7. Comparison of Age-related Macular Degeneration Treatments Trials Research Group, Maguire MG, Martin DF, Ying GS, Jaffe GJ, Daniel E, et al. Five-year outcomes with anti-vascular endothelial growth factor treatment of neovascular age-related macular degeneration: the comparison of age-related macular degeneration treatments trials. Ophthalmology. 2016;123:1751-61.

8. Schmidt-Erfurth U, Waldstein SM. A paradigm shift in imaging biomarkers in neovascular age-related macular degeneration. Prog Retinal Eye Res. 2016;50:1-24.

9. Jaffe GJ, Martin DF, Toth CA, Daniel E, Maguire MG, Ying GS, et al. Macular morphology and visual acuity in the comparison of age-related macular degeneration treatments trials. Ophthalmology. 2013;120:1860-70.

10. Simader C, Ritter M, Bolz M, Deak G, Sponer U, Golbaz I, et al. Morphologic parameters relevant for visual outcome during antiangiogenic therapy of neovascular age-related macular degeneration. Ophthalmology. 2014;121:1237-45.

11. Waldstein SM, Simader C, Staurenghi G, Chong NV, Mitchell P, Schmidt-Erfurth, et al. Morphology and visual acuity in aflibercept and ranibizumab therapy for neovascular age-related macular degeneration in the VIEW trials. Ophthalmology. 2016;123:1521-9.

12. Cheung CMG, Grewal DS, Teo KYC, Gan A, Mohla A, Chakravarthy $\mathrm{U}$, et al. The evolution of fibrosis and atrophy and their relationship with visual outcomes in Asian subjects with neovascular age-related macular degeneration. Ophthalmol Retina. 2020;3:1045-55.

13. Sarraf D, London NJ, Khurana RN, Dugel PU, Gune S, Hill L, et al. Ranibizumab treatment for pigment epithelial detachment secondary to neovascular age-related macular degeneration: post hoc analysis of the HARBOR study. Ophthalmology. 2016;123:2213-24.

14. Waldstein SM, Wright J, Warburton J, Margaron P, Simader C, Schmidt-Erfurth U. Predictive value of retinal morphology for visual acuity outcomes of different ranibizumab treatment regimens for neovascular AMD. Ophthalmology. 2016;123:60-9.

15. Waldstein SM, Simader C, Staurenghi G, Chong NV, Mitchell P, Jaffe GJ, et al. Morphology and visual acuity in aflibercept and ranibizumab therapy for neovascular age-related macular degeneration in the VIEW trials. Ophthalmology. 2016;123:1521-9.

16. Simader C, Ritter M, Bolz M, Deák GG, Mayr-Sponer U, Golbaz I, et al. Morphologic parameters relevant for visual outcome during anti-angiogenic therapy of neovascular age-related macular degeneration. Ophthalmology. 2014;121:1237-45.

17. Schmidt-Erfurth U, Waldstein SM, Deák GG, Kundi M, Simader C. Pigment epithelial detachment followed by retinal cystoid degeneration leads to vision loss in treatment of neovascular agerelated macular degeneration. Ophthalmology. 2015;122:822-32.

18. Ritter M, Simader C, Bolz M, Deák GG, Mayr-Sponer U, Sayegh $\mathrm{R}$, et al. Intraretinal cysts are the most relevant prognostic biomarker in neovascular age-related macular degeneration independent of the therapeutic strategy. $\mathrm{Br} \mathrm{J}$ Ophthalmol. 2014;98:1629-35. 
19. Inoue M, Arakawa A, Yamane S, Kadonosono K. Variable response of vascularized pigment epithelial detachments to ranibizumab based on lesion subtypes, including polypoidal choroidal vasculopathy. Retina. 2013;33:990-7.

20. Lalwani GA, Rosenfeld PJ, Fung AE, Dubovy SR, Michels S, Feuer W, et al. A variable-dosing regimen with intravitreal ranibizumab for neovascular age-related macular degeneration: year 2 of the PrONTO Study. Am J Ophthalmol. 2009;148:43-58.e1.

21. Fung AE, Lalwani GA, Rosenfeld PJ, Dubovy SR, Michels S, Feuer WJ, et al. An optical coherence tomography-guided, variable dosing regimen with intravitreal ranibizumab (Lucentis) for neovascular age-related macular degeneration. Am J Ophthalmol. 2007;143:566-83.

22. Lai TT, Hsieh YT, Yang CM, Ho TC, Yang CH. Biomarkers of optical coherence tomography in evaluating the treatment outcomes of neovascular age-related macular degeneration: a realworld study. Sci Rep. 2019;9:529.

23. Leung KFC, Downes SM, Chong V. A retrospective analysis of the effect of subretinal hyper-reflective material and other morphological features of neovascular age-related macular degeneration on visual acuity outcomes in eyes treated with intravitreal aflibercept over one year. Vision. 2018;2:5.

24. Tyagi P, Juma Z, Hor YK, Scott NW, Ionean A, Santiago C. Clinical response of pigment epithelial detachment associated with neovascular age-related macular degeneration in switching treatment from Ranibizumab to Aflibercept. BMC Ophthalmol. 2018;18:148.

25. Balaskas K, Karampelas M, Horani M, Hotu O, Keane P, Aslam T. Quantitative analysis of pigment epithelial detachment response to different anti-vascular endothelial growth factor agents in wet age-related macular degeneration. Retina. 2017;37:1297-304.

26. Suzuki M, Nagai N, Izumi-Nagai K, Shinoda H, Koto T, Uchida $\mathrm{A}$, et al. Predictive factors for non-response to intravitreal ranibizumab treatment in age-related macular degeneration. $\mathrm{Br} \mathrm{J}$ Ophthalmol. 2014;98:1186-91.

27. Ersoy L, Ristau T, Kirchhof B, Liakopoulos S. Response to antiVEGF therapy in patients with subretinal fluid and pigment epithelial detachment on spectral-domain optical coherence tomography. Graefes Arch Clin Exp Ophthalmol. 2014;252:889-97.

28. Ristau T, Hillebrand S, Smailhodzic D, Walsh AC, Kirchhof B, Sadda SR, et al. Prognostic factors for long term visual acuity outcome after ranibizumab therapy in patients with neovascular age-related macular degeneration. J Clin Exp Ophthalmol. 2013; 4:264.

29. Baba T, Kitahashi M, Kubota-Taniai M, Oshitari T, Yamamoto S. Two-year course of subfoveal pigment epithelial detachment in eyes with age-related macular degeneration and visual acuity better than 20/40. Ophthalmologica. 2012;228:102-9.

30. Freeman WR, Kozak I, Yuson RM, Nigam N, Cheng L, Mojana F. Prognostic implications of pigment epithelial detachment in bevacizumab (avastin)-treated eyes with age-related macular degeneration and choroidal neovascularization. Retina. 2011;31:1812-8.

31. Mariani A, Deli A, Ambresin A, Mantel I. Characteristics of eyes with secondary loss of visual acuity receiving variable dosing ranibizumab for neovascular age-related macular degeneration. Graefes Arch Clin Exp Ophthalmol. 2011;249:1635-42.

32. Singh RP, Fu EX, Smith SD, Williams DR, Kaiser PK. Predictive factors of visual and anatomical outcome after intravitreal bevacizumab treatment of neovascular age-related macular degeneration: an optical coherence tomography study. Br J Ophthalmol. 2009;93:1353-8.

33. Bolz M, Michels S, Geitzenauer W, Prager F, Schmidt-Erfurth U. Effect of systemic bevacizumab therapy on retinal pigment epithelial detachment. Br J Ophthalmol. 2007;91:785-9.

34. Chen E, Kaiser RS, Vander JF. Intravitreal bevacizumab for refractory pigment epithelial detachment with occult choroidal neovascularization in age-related macular degeneration. Retina. 2007;27:445-50.

35. Cheung CMG, Bhargava M, Laude A, Koh ACh, Xiang L, Wong $\mathrm{D}$, et al. Asian age-related macular degeneration phenotyping study: rationale, design and protocol of a prospective cohort study. Clin Exp Ophthalmol. 2012;40:727-35.

36. Iijima $\mathrm{H}$, Iida $\mathrm{T}$, Imai $\mathrm{M}$, Gohdo $\mathrm{T}$, Tsukahara $\mathrm{S}$. Optical coherence tomography of orange-red subretinal lesions in eyes with idiopathic polypoidal choroidal vasculopathy. Am J Ophthalmol. 2000;129:21-6.

37. Giovannini A, Amato G, Mariotti C, Scassellati-Sforzolini B. Optical coherence tomography in the assessment of retinal pigment epithelial tear. Retina. 2000;20:37-40.

38. Sarraf D, Reddy S, Chiang A, Yu F, Jain A. A new grading system for retinal pigment epithelial tears. Retina. 2010;30: 1039-45.

39. Pang CE, Messinger JD, Zanzottera EC, Freund KB, Curcio CA. The onion sign in neovascular age-related macular degeneration represents cholesterol crystals. Ophthalmology. 2015;122: 2316-26.

40. Tan ACS, Simhaee D, Balaratnasingam C, Dansingani KK, Yannuzzi LA. A perspective on the nature and frequency of pigment epithelial detachments. Am J Ophthalmol. 2016;172: $13-27$.

41. Clemens CR, Eter N. Retinal pigment epithelium tears: risk factors, mechanism and therapeutic monitoring. Ophthalmologica. 2016;235:1-9.

42. Khanani AM, Eichenbaum D, Schlottmann PG, Tuomi L, Sarraf D. Optimal management of pigment epithelial detachments in eyes with neovascular age-related macular degeneration. Retina. 2018;38:2103-17.

43. Tsai ASH, Cheung N, Gan ATL, Jaffe GJ, Sivaprasad S, Wong TY, et al. Retinal angiomatous proliferation. Surv Ophthalmol. 2017;62:462-92.

44. Kumar A, Kumawat D, Sundar MD, Gagrani M, Gupta B, Roop P, et al. Polypoidal choroidal vasculopathy: a comprehensive clinical update. Ther Adv Ophthalmol. 2019;11: 2515841419831152.

45. De Salvo G, Vaz-Pereira S, Keane PA, Tufail A, Liew G. Sensitivity and specificity of spectral-domain optical coherence tomography in detecting idiopathic polypoidal choroidal vasculopathy. Am J Ophthalmol. 2014;158:1228-38.e1. 